\title{
Наногетероструктуры с улучшенными параметрами для быстродействующих и высокоэффективных плазмон-поляритонных светодиодов Шоттки
}

\author{
(C) Н.В. Байдусь ${ }^{+*}$, В.А. Кукушкин ${ }^{+\ddagger}$, Б.Н. Звонков*, С.М. Некоркин* \\ + Нижегородский государственный университет им. Н.И. Лобачевского, \\ 603950 Нижний Новгород, Россия \\ * Научно-исследовательский физико-технический институт \\ Нижегородского государственного университета им. Н.И. Лобачевского, \\ 603950 Нижний Новгород, Россия \\ ¥ Институт прикладной физики Российской академии наук, \\ 603950 Нижний Новгород, Россия \\ E-mail: vakuk@appl.sci-nnov.ru
}

(Получена 26 апреля 2016 г. Принята к печати 10 мая 2016 г.)

В результате теоретического и экспериментального анализа найдены параметры гетероструктур с квантовыми точками InAs в матрице GaAs, обеспечивающие создание на таких структурах быстродействующих и высокоэффективных плазмон-поляритонных светодиодов Шоттки для ближней инфракрасной области. Квантовые точки должны располагаться на сильно легированном (вплоть до концентрации легирующей примеси $10^{19} \mathrm{~cm}^{-3}$ ) буферном слое GaAs и отделяться от металла тонким (толщиной 10-30 нм) нелегированным покровным слоем GaAs. Граница раздела металла (например, золота) с GaAs обеспечит эффективное рассеяние поверхностных плазмон-поляритонов в обычные фотоны в случае, если она имеет неоднородности в виде заполненных металлом впадин в GaAs с характерным размером $\sim 30$ нм и поверхностной концентрацией, превышающей $10^{10} \mathrm{~cm}^{-2}$.

\section{1. Введение}

Создание быстродействующих и высокоэффективных, т. е. обладающих высоким коэффициентом полезного действия (кпд), светодиодов Шоттки ближнего инфракрасного диапазона частот является актуальной задачей современной полупроводниковой оптоэлектроники в связи с их востребованностью в телекоммуникационных и информационных приложениях. Интересным объектом для исследования в качестве их активной области являются квантовые точки (КТ) благодаря высокому ограничивающему потенциалу и, как следствие, способности сохранять высокую интенсивность люминесценции при комнатной температуре [1].

Для повышения быстродействия таких устройств необходимо обеспечить быстрое гашение фотолюминесценции КТ при выключении токовой накачки. В принципе этого можно достичь, вводя в структуру дополнительные центры безызлучательной рекомбинации находящихся в КТ электронов и дырок. Однако этот подход приведет к резкому уменьшению кпд основанного на такой структуре светодиода Шоттки. Имеется и другой путь повышения быстродействия последнего без существенного снижения его кпд. Он заключается в уменьшении (до нескольких десятков нанометров) расстояния между слоем КТ и металлическим контактом Шоттки. В результате при рекомбинации электронов и дырок в КТ будут излучаться не только обычные фотоны, но и поверхностные плазмон-поляритоны, представляющие собой локализованные на границе раздела полупроводника и металла (т. е. двух сред с разными знаками действительных частей диэлектрических проница- емостей) самосогласованные колебания поляризации и электромагнитного поля [2]. Это приведет к проявлению так называемого эффекта Парселла [3] - ускорению гашения фотолюминесценции КТ при выключении токовой накачки. С другой стороны, поверхностные плазмонполяритоны вследствие рассеяния на шероховатостях границы полупроводника и металла превращаются в обычные фотоны, которые затем свободно покидают структуру. В результате кпд основанного на такой структуре светодиода Шоттки практически не снизится.

Ранее были исследованы основные закономерности фотолюминесценции сферических КТ, расположенных вблизи плоского металлического контакта [4]. Нами была показана возможность возбуждения электролюминесценции от слоя KT InAs в GaAs в прямо смещенном диоде Шоттки, в котором этот слой располагался относительно близко (на расстоянии 10-30 нм) от металлического контакта [5].

Целью настоящей работы является теоретическое и экспериментальное определение параметров гетероструктур с КТ, обеспечивающих интенсивное возбуждение поверхностных плазмон-поляритонов и их рассеяние в обычные фотоны для создания на основе таких структур быстродействующих и высокоэффективных светодиодов Шоттки ближнего инфракрасного диапазона частот.

\section{2. Улучшенные параметры квантовых точек}

Поверхностным плазмон-поляритоном называют электромагнитную волну, локализованную вблизи поверх- 
ности раздела двух сред с разными знаками действительных частей диэлектрических проницаемостей [2]. На практике одной из таких сред может быть металл $\left(\operatorname{Re} \varepsilon_{1}<0\right)$, а второй - полупроводник $\left(\operatorname{Re} \varepsilon_{2}>0\right)-$ cм. рис. 1 . В рассматриваемом нами диапазоне частот $\omega$ модуль действительной части $\varepsilon_{1}$ значительно превышает модуль ее мнимой части, которой мы поэтому будем пренебрегать. ${ }^{1}$ Для вычисления $\varepsilon_{1}$ мы используем модель Друде [6]. Согласно хорошо известным формулам [2], в случае достаточно толстого слоя металла 1 закон дисперсии поверхностного плазмон-поляритона дается формулой $k_{\|}=(\omega / c) \sqrt{\varepsilon_{1} \varepsilon_{2} /\left(\varepsilon_{1}+\varepsilon_{2}\right)}$, где $k_{\|}$- величина параллельной поверхности раздела металл-полупроводник компоненты его волнового вектора, $c$ - скорость света в вакууме. Для рассматриваемых в настоящей работе частот и материалов $\left|\operatorname{Re} \varepsilon_{1}\right|>\operatorname{Re} \varepsilon_{2}$ (что обеспечивает положительность действительной части подкоренного выражения в формуле для $k_{\|}$), а затухание поверхностного плазмон-поляритона с расстоянием определяется в основном мнимой частью $\varepsilon_{1}$. Проекции волнового вектора поверхностного плазмон-поляритона на ортогональную плоскости структуры ось $x$ в металле и полупроводнике, $k_{x 1}$ и $k_{x 2}$ соответственно (см. pис. 1), даются формулами $k_{x 1}=-i \omega \varepsilon_{1} /\left[c \sqrt{-\left(\varepsilon_{1}+\varepsilon_{2}\right)}\right]$ и $k_{x 2}=-i \omega \varepsilon_{2} /\left[c \sqrt{-\left(\varepsilon_{1}+\varepsilon_{2}\right)}\right]$.

Оператор вектор-потенциала поверхностного плазмон-поляритона в кулоновской калибровке имеет вид

$$
\hat{\mathbf{A}}(\mathbf{r}, t)=\sum_{\mathbf{k}_{\|}}\left(\hat{a}_{\mathbf{k}_{\|}} \mathbf{A}_{\mathbf{k}_{\|}}+\hat{a}_{\mathbf{k}_{\|}}^{+} \mathbf{A}_{\mathbf{k}_{\|}}^{*}\right)
$$

где $\hat{a}_{\mathbf{k}_{\|}}, \hat{a}_{\mathbf{k}_{\|}}^{+}-$операторы уничтожения и рождения поверхностного плазмон-поляритона с параллельной поверхности раздела компонентой волнового вектора $\mathbf{k}_{\|}$ соответственно, суммирование ведется по всем $\mathbf{k}_{\|}$, удовлетворяющим двумерным периодическим граничным условиям Борна-Кармана [6],

$$
\begin{aligned}
\mathbf{A}_{\mathbf{k}_{\|}}(x>0)= & A_{0}\left(-k_{\|} \mathbf{x}^{0} / k_{x 1}+\mathbf{k}_{\|} / k_{\|}\right) \\
& \times \exp \left(-i \omega t+i \mathbf{k}_{\|} \mathbf{r}_{\|}+i k_{x 1} x\right), \\
\mathbf{A}_{\mathbf{k}_{\|}}(x<0)= & A_{0}\left(-k_{\|} \mathbf{x}^{0} / k_{x 2}+\mathbf{k}_{\|} / k_{\|}\right) \\
\times & \exp \left(-i \omega t+i \mathbf{k}_{\|} \mathbf{r}_{\|}+i k_{x 2} x\right), \\
A_{0}=\frac{\sqrt{8 \pi c \hbar}}{\sqrt{S}\left[-\left(\varepsilon_{1}+\varepsilon_{2}\right)\right]^{1 / 4}\left\{\left[\left(1-\varepsilon_{1} / \varepsilon_{2}\right) \omega \varepsilon_{2}^{\prime}-2 \varepsilon_{1}\right] / \varepsilon_{2}-\right.} & \left.-\left[\left(1-\varepsilon_{2} / \varepsilon_{1}\right) \omega \varepsilon_{1}^{\prime}-2 \varepsilon_{2}\right] / \varepsilon_{1}\right\}^{1 / 2}
\end{aligned}
$$

где $\hbar$ - постоянная Планка, $S$ - площадь поверхности раздела металл-диэлектрик, штрих означает производную по частоте. При этом слой металла 1 (рис. 1) считается достаточно толстым (т. е. имеющим толщину,

\footnotetext{
${ }^{1}$ Однако этого нельзя делать при вычислении определяемого мнимой частью $\varepsilon_{1}$ коэффициента затухания поверхностного плазмонполяритона.
}

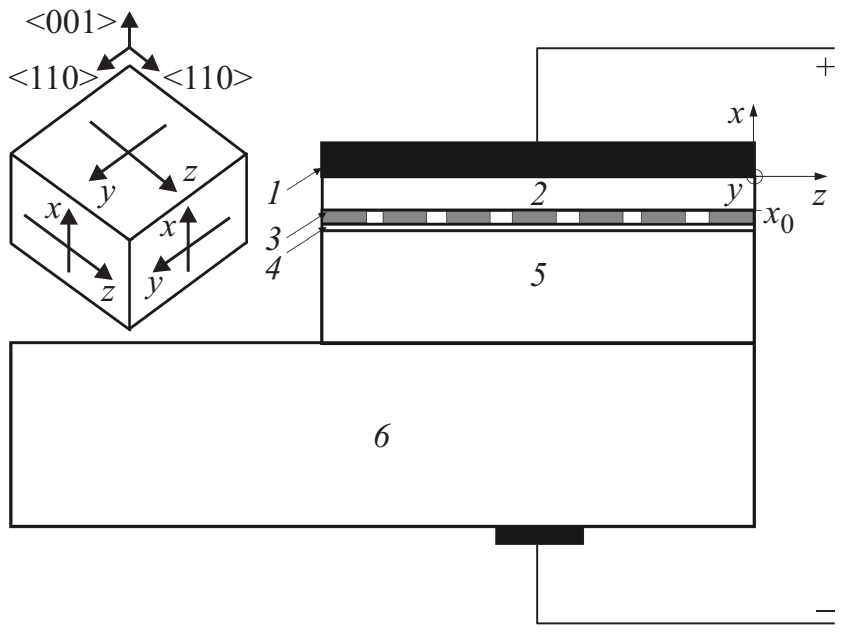

Рис. 1. Схема плазмон-поляритонного светодиода Шоттки и используемая в тексте декартова система координат (также указаны соответствующие ее осям кристаллографические направления): 1 - металл, 2 - покровный слой, 3 - КТ, 4 - спейсер, 5 - буферный слой, 6 - подложка.

бо́льшую масштаба $1 / \operatorname{Im} k_{x 1}$ спадания поля поверхностного плазмон-поляритона в металле, который для длины волны излучения 1 мкм равняется $\sim 20 \mathrm{Hм}$ ). Величина нормировочного множителя $A_{0}$ выбрана из условия, чтобы энергия одного поверхностного плазмон-поляритона равнялась $\hbar \omega$. Эта энергия вычисляется с учетом частотной дисперсии диэлектрических проницаемостей граничащих сред [7-9] при пренебрежении их мнимыми частями по сравнению с действительными.

Используя стандартное выражение для нерелятивистского гамильтониана взаимодействия электрона с электромагнитным полем и оставляя в нем лишь резонансные члены, из уравнения Шредингера легко получить систему уравнений для амплитуд состояний, отвечающих возбужденной КТ (т.е. КТ, в которой находится один экситон) и отсутствию поверхностных плазмонполяритонов $\left(c_{1}\right)$ и невозбужденной КТ (т.е. КТ без экситона) и одного поверхностного плазмон-поляритона с параллельной поверхности раздела компонентой волнового вектора $\mathbf{k}_{\|}\left(c_{0 \mathbf{k}_{\|}}\right)$:

$$
\begin{gathered}
i \hbar \dot{c}_{1}=-\frac{e}{m c} \sum_{\mathbf{k}_{\|}} \mathbf{A}_{\mathbf{k}_{\|}}\left(x=x_{0}\right) \mathbf{p}_{10} \exp \left(i \omega_{0} t\right) c_{0 \mathbf{k}_{\|}}, \\
i \hbar \dot{c}_{0 \mathbf{k}_{\|}}=-\frac{e}{m c} \mathbf{A}_{\mathbf{k}_{\|}}^{*}\left(x=x_{0}\right) \mathbf{p}_{01} \exp \left(-i \omega_{0} t\right) c_{1},
\end{gathered}
$$

где точка обозначает дифференцирование по времени, $x_{0}<0, x-$ координата плоскости КТ (рис. 1), $e-$ величина элементарного заряда $(e>0), m-$ масса свободного электрона, $\mathbf{p}_{10}=\mathbf{p}_{01}^{*}-$ матричный элемент оператора импульса электрона между его основным (энергия $E_{0}$ ) и возбужденным (энергия $E_{1}$ ) состояниями в КТ, $\omega_{0} \equiv\left(E_{1}-E_{0}\right) / \hbar$ - частота перехода. 
Из этой системы в приближении Вигнера-Вайскопфа [10] получается уравнение для $c_{1}$ :

$$
\begin{aligned}
\dot{c}_{1}= & -\left(\frac{e}{m c \hbar}\right)^{2} \pi c_{1} \sum_{\mathbf{k}_{\|}} \delta\left(\omega-\omega_{0}\right) \exp \left[-2 \operatorname{Im}\left(k_{x 2}\right) x_{0}\right]\left|A_{0}\right|^{2} \\
& \times\left|-\frac{k_{\|} p_{01 x}}{k_{x 2}}+\frac{\mathbf{p}_{01} \mathbf{k}_{\|}}{\mathbf{k}_{\|}}\right|^{2} \equiv-\frac{c_{1} \Gamma_{\mathrm{pl}}}{2},
\end{aligned}
$$

где $p_{01 x}-$ проекция $\mathbf{p}_{01}$ на ортогональную плоскости структуры ось $x$ (см. рис. 1). Суммирование по $\mathbf{k}_{\|}$ заменим интегрированием с помощью формулы

$$
\sum_{\mathbf{k}_{\|}} \rightarrow \int_{0}^{+\infty} \int_{0}^{2 \pi} \frac{S k_{\|}}{(2 \pi)^{2}} d \phi d k_{\|}
$$

где $\phi$ - угол в плоскости структуры, характеризующий направление $\mathbf{k}_{\|}, \Gamma_{\mathrm{pl}}$ - скорость возбуждения КТ поверхностных плазмон-поляритонов. Выполняя интегрирование по $\phi$ и $k_{\|}$с использованием выражения для закона дисперсии поверхностного плазмон-поляритона, легко показать, что

$$
\begin{aligned}
& \Gamma_{\mathrm{pl}}=\left(\frac{e}{m c \hbar}\right)^{2} \frac{S \exp \left[-2 \operatorname{Im}\left(k_{x 2}\right) x_{0}\right]\left|A_{0}\right|^{2} \omega_{0}}{c^{2}\left(\varepsilon_{1}+\varepsilon_{2}\right)} \\
& \quad \times\left(\varepsilon_{1} \varepsilon_{2}+\frac{\omega_{0}}{2} \frac{\varepsilon_{1}^{\prime} \varepsilon_{2}^{2}+\varepsilon_{2}^{\prime} \varepsilon_{1}^{2}}{\varepsilon_{1}+\varepsilon_{2}}\right)\left(\frac{k_{\|}^{2}\left|p_{01 x}\right|^{2}}{\left|k_{x 2}\right|^{2}}+\frac{\left|\mathbf{p}_{01 \|}\right|^{2}}{2}\right),
\end{aligned}
$$

где все величины берутся при $\omega=\omega_{0}$ и $\mathbf{p}_{01 \|}-$ компонента $\mathbf{p}_{01}$ в плоскости структуры. Таким образом, скорость возбуждения КТ поверхностных плазмон-поляритонов $\Gamma_{\mathrm{pl}}$ быстро растет при приближении модуля отрицательной диэлектрической проницаемости металла к положительной диэлектрической проницаемости полупроводника. При точном их совпадении величина $\Gamma_{\mathrm{pl}}$ должна, однако, оставаться конечной, что обеспечивается не учтенными здесь мнимыми частями диэлектрических проницаемостей $\varepsilon_{1}$ и $\varepsilon_{2}$.

В уравнения (2), (3) и (4) не включена возможность перехода КТ из возбужденного в основное состояние с излучением не поверхностного плазмон-поляритона, а обычного фотона. Для ее учета в указанные уравнения необходимо добавить соответствующие слагаемые и провести вычисления в рамках стандартного метода Вигнера-Вайскопфа [10] с использованием обычных формул квантовой электродинамики сплошных сред $[8]{ }^{2}$ В результате получаем, что скорость излучения обычных фотонов дается формулой $[8,10]$

$$
\Gamma_{\mathrm{ph}} \equiv 4 e^{2} \sqrt{\varepsilon_{2}} \omega_{0}\left|\mathbf{p}_{01}\right|^{2} /\left(3 \hbar c^{3} m^{2}\right)
$$

\footnotetext{
2 При этом для оценок мы пренебрегаем наличием металла, т.е. считаем, что КТ излучают фотоны так, как если бы они находились в безграничной однородной изотропной среде с диэлектрической проницаемостью $\varepsilon_{2}$.
}

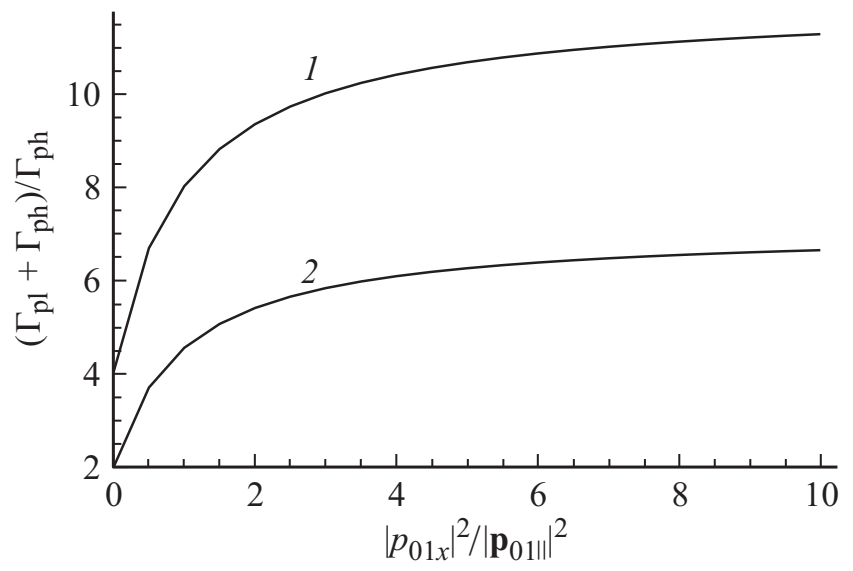

Рис. 2. Зависимости $\left(\Gamma_{\mathrm{pl}}+\Gamma_{\mathrm{ph}}\right) / \Gamma_{\mathrm{ph}}$ от $\left|p_{01 x}\right|^{2} /\left|\mathbf{p}_{01 \|}\right|^{2}$ при толщине покровного слоя 20 нм для вакуумных длин волн излучения 0.91 (1) и 1.2 мкм (2).

Степень влияния границы металл-полупроводник на излучательное время жизни КТ в возбужденном состоянии удобно характеризовать отношением $\left(\Gamma_{\mathrm{pl}}+\Gamma_{\mathrm{ph}}\right) / \Gamma_{\mathrm{ph}}$ скорости их перехода в основное состояние при наличии металла к аналогичной величине в его отсутствие. Поскольку $\left|\mathbf{p}_{01}\right|^{2} \equiv\left|p_{01 x}\right|^{2}+\left|\mathbf{p}_{01 \|}\right|^{2}$, видно, что матричные элементы оператора импульса электрона между его основным и возбужденным состояниями в КТ входят в это отношение только в виде комбинации $\left|p_{01 x}\right|^{2} /\left|\mathbf{p}_{01 \|}\right|^{2}$. Зависимости $\left(\Gamma_{\mathrm{pl}}+\Gamma_{\mathrm{ph}}\right) / \Gamma_{\mathrm{ph}}$ от $\left|p_{01 x}\right|^{2} /\left|\mathbf{p}_{01 \|}\right|^{2}$ для различных длин волн излучения ближнего инфракрасного диапазона в пренебрежении частотной дисперсией диэлектрической проницаемости GaAs в нем (т.е. при $\left.\varepsilon_{2}^{\prime}=0\right)$ приведены на рис. 2. Рост $\left(\Gamma_{\mathrm{pl}}+\Gamma_{\mathrm{ph}}\right) / \Gamma_{\mathrm{ph}} \mathrm{c}$ увеличением $\left|p_{01 x}\right|^{2} /\left|\mathbf{p}_{01 \|}\right|^{2}$ объясняется тем, что, согласно (1), компонента вектор-потенциала (следовательно, и электрического поля) поверхностного плазмонполяритона в перпендикулярном плоскости структуры направлении в $k_{\|} / k_{x 2} \approx 2$ раза больше, чем компонента его вектор-потенциала (следовательно, и электрического поля) в плоскости структуры. Поэтому КТ с большой проекцией матричного элемента оператора импульса электрона между его основным и возбужденным состояниями на перпендикулярное плоскости структуры направление излучают поверхностные плазмон-поляритоны более эффективно.

Поскольку излучение КТ обычных фотонов является дипольным, то в отсутствие металла отношение интенсивностей люминесценции КТ с двумя ортогональными поляризациями равно отношению квадратов модулей проекций $\mathbf{p}_{01}$ на направления этих поляризаций. Это открывает возможность экспериментального определения $\left|p_{01 x}\right|^{2} /\left|\mathbf{p}_{01 \|}\right|^{2}$ для КТ путем поляризационных измерений интенсивности излучения, выходящего через два взаимно ортогональных торца неметаллизированной гетероструктуры с КТ или через один ее торец и лицевую поверхность. 
Отношения квадратов модулей проекций р 01 на оси декартовой системы координат для КТ в образцах с разными степенями легирования буферного слоя

\begin{tabular}{c|c|c|c|c|c|c}
\hline $\begin{array}{c}\text { Номер } \\
\text { структуры }\end{array}$ & $\begin{array}{c}\text { Степень } \\
\text { легирования, см }\end{array}$ & $\frac{\left|p_{01 z}\right|^{2}}{\left|p_{01 y}\right|^{2}}$ & $\frac{\left|p_{01 x}\right|^{2}}{\left|p_{01 y}\right|^{2}}$ & $\frac{\left|p_{01 x}\right|^{2}}{\left|p_{01 z}\right|^{2}}$ & $\frac{\left|p_{01 x}\right|^{2}}{\left|p_{01 y}\right|^{2}+\left|p_{01 z}\right|^{2}}$ & $\begin{array}{c}\frac{\Gamma_{\mathrm{pl}}+\Gamma_{\mathrm{ph}}}{\Gamma_{\mathrm{ph}}}, \mathrm{pacчет} \\
(\text { при } 77 \mathrm{~K})\end{array}$ \\
\hline 7806 & $10^{18}$ & 1.23 & 1.05 & 0.8 & 0.46 & 3.61 \\
7923 & $5 \cdot 10^{17}$ & 1.43 & 2.2 & 1.54 & 0.9 & 4.44 \\
7932 & $10^{19}$ & 1.24 & 2 & 1.62 & 0.89 & 4.43 \\
7878 & $10^{17}$ & 1.5 & 1.39 & 0.89 & 0.6 & 3.28 \\
$7878 \mathrm{c} \mathrm{Au}$ & $10^{17}$ & 1.14 & 2.47 & 2.16 & 1.15 &
\end{tabular}

Ранее [11] были экспериментально определены необходимые для создания КТ с большим отношением $\left|p_{01 x}\right|^{2} /\left|\mathbf{p}_{01 \|}\right|^{2}$ условия: тонкий покровный слой (т.е. слой, отделяющий слой КТ от поверхности образца) и сильное легирование буферного (т.е. выращенного на подложке GaAs и расположенного под КТ) слоя GaAs при отсутствии легирования покровного слоя. Для продолжения этих исследований методом МОС-гидридной эпитаксии (газофазной эпитаксии из металлорганических соединений) нами были изготовлены гетероструктуры с KT InAs/GaAs. Структуры, КТ в которых обладали большим отношением $\left|p_{01 x}\right|^{2} /\left|\mathbf{p}_{01 \|}\right|^{2}$, состояли из выращенного на подложке GaAs 6 (см. рис. 1) сильно легированного (до концентраций $10^{17}-10^{19} \mathrm{~cm}^{-3}$ ) буферного слоя 5 толщиной 0.5-1 мкм, тонкого (толщина 5 нм) нелегированного спейсера GaAs 4, одиночного слоя КТ 3 и нелегированного покровного слоя 2 толщиной 20 нм (исключением является структура 7878 (см. таблицу), для которой толщина покровного слоя равнялась 30 нм). Металл 1 в виде полупрозрачной (толщина 10 нм) пленки золота наносился только на часть структуры 7878. Исследовались структуры с $n$ - и $p$-типом проводимости. Оптическая накачка осуществлялась сфокусированным лучом гелий-неонового лазера с мощностью $30 \mathrm{MBT}$ с лицевой поверхности гетероструктуры. Излучение собиралось линзой с лицевой поверхности и с двух взаимно ортогональных сколотых торцов структуры. Интенсивности излучения с двумя взаимно ортогональными линейными поляризациями измерялись с помощью призмы Глана-Тейлора, размещенной перед входной щелью решеточного монохроматора МДР-3. Измерения производились при $77 \mathrm{~K}$ с применением германиевого фотодиода, их результаты приведены на рис. $3, a, b$ и в таблице.

Для всех структур, как и для структуры 7878 (рис. 3, $a$ и $b$ ), максимум интенсивности фотолюминесценции при $77 \mathrm{~K}$ лежал в области $1.15-1.18$ мкм (1.05-1.08 эВ) и был обусловлен излучением КТ. Из сравнения рис. $3, a$ и $b$ видно, что пленка золота значительно увеличивает интенсивность фотолюминесценции с ортогональной плоскости структуры поляризацией. Сравнение структур 7923, 7932 и 7878 показывает, что увеличение уровня легирования буферного слоя (и, следовательно, напряженности электрического поля в слое КТ) приводит к росту степени ортогональной плоскости структуры поляризации фотолюминесценции $\left|p_{01 x}\right|^{2} /\left|p_{01 y}\right|^{2}$, которая достигает значений $2.0-2.2$. В случае отсутствия электрического поля в слое КТ (т.е. для однородно сильнолегированного образца 7806) отношения $\left|p_{01 x}\right|^{2} /\left|p_{01 y}\right|^{2}$ и $\left|p_{01 x}\right|^{2} /\left|p_{01 z}\right|^{2}$ близки к единице, т. е. фотолюминесценция КТ поляризована примерно изотропно. В последних двух строках таблицы приведены данные по поляризации
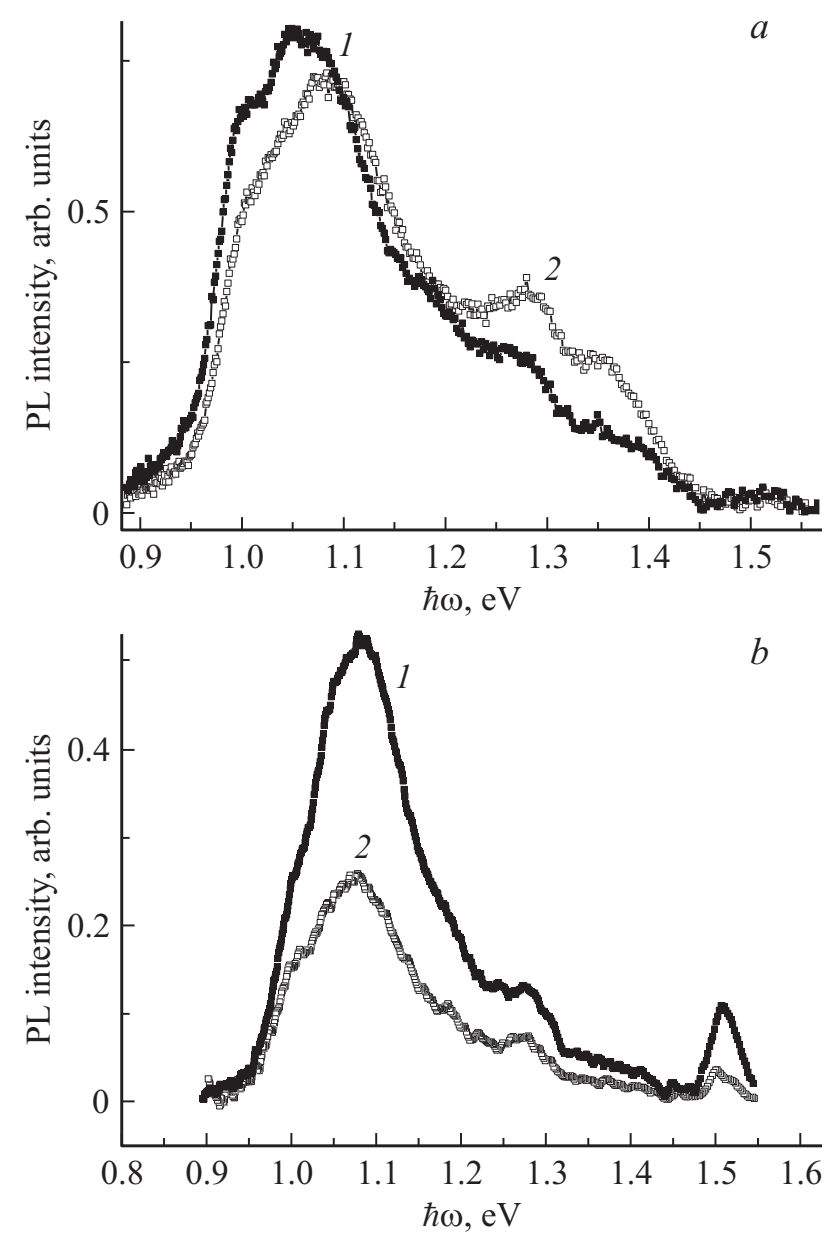

Рис. 3. Спектры фотолюминесценции (PL) структуры 7878, наблюдаемой с ее скола при $77 \mathrm{~K}$, для электрического поля, ортогонального плоскости структуры (1), и для электрического поля в плоскости структуры (2). a - без золота, $b-$ с полупрозрачным слоем золота толщиной 10 нм. 
фотолюминесценции образца 7878 со свободной поверхностью и с полупрозрачной (толщина 10 нм) пленкой золота. Видно, что вследствие изменения конфигурации и напряженности встроенного электрического поля, а также возбуждения поверхностных плазмон-поляритонов пленка золота увеличивает ортогональную плоскости структуры компоненту поляризации излучения. Для проведения эксперимента с покрытием поверхности наногетероструктуры полупрозрачной пленкой золота был выбран образец 7878 с наименьшим из использованных уровнем легирования буферного слоя, так как до покрытия золотом этот образец характеризовался малой степенью ортогональной плоскости структуры поляризации фотолюминесценции КТ и поэтому после покрытия золотом относительное увеличение степени этой поляризации для данного образца было весьма значительным.

Возможное объяснение наблюдавшейся аномально большой ортогональной плоскости структуры поляризации фотолюминесценции КТ состоит в следующем. Как известно, поляризация излучения КТ определяется видом дырок, участвующих в излучательной рекомбинации с электронами. В стандартном случае это тяжелые дырки, что и приводит к тому, что вектор электрического поля излучения лежит в плоскости структуры. Преобладание ортогональной плоскости структуры поляризации свидетельствует о доминирующем участии в излучательной рекомбинации с электронами легких дырок. Для объяснения этого эффекта можно предложить следующую модель. Расчеты [12] упругих напряжений и зонной структуры гетеросистемы с двумя слоями квантовых точек показывают, что слой InAs испытывает напряжения сжатия, а слой $\mathrm{GaAs}$ - растягивающие напряжения, в результате чего подзона легких дырок в GaAs поднимается по энергии выше подзоны тяжелых дырок GaAs, но все же остается ниже энергии подзоны тяжелых дырок в KT InAs. Сильное электрическое поле увеличивает интеграл перекрытия волновых функций электронов в КT InAs и легких дырок в GaAs. B peзультате увеличивается вероятность излучения фотонов с ортогональной к плоскости структуры поляризацией за счет непрямых (в пространстве) оптических переходов.

\section{3. Улучшенные параметры неоднородностей границы металл-полупроводник}

Длина поглощения поверхностных плазмон-поляритов (по интенсивности) за счет омической диссипации в металле равняется $1 /\left(2 \operatorname{Im} k_{\|}\right)$и на границе GaAs c золотом для вакуумной длины волны излучения 1 мкм составляет $\sim 7$ мкм, т. е. величину, много меньшую латеральных размеров структуры. Поэтому поверхностные плазмон-поляритоны, возбужденные большей частью КТ, будут поглощаться в самой же структуре, не дойдя до ее торцов, где они могли бы превратиться в обычные фотоны и излучиться в окружающее пространство. В результате кпд рассматриваемого плазмон-поляритонного диода Шоттки будет низким. Для его повышения необходимо преобразовать поверхностные плазмонполяритоны в обычные фотоны на длине, существенно меньшей длины их поглощения. Одним из возможных механизмов такого преобразования является рассеяние поверхностных плазмон-поляритонов в обычные фотоны на неоднородностях границы металл-полупроводник. Настоящий раздел посвящен подробному рассмотрению этого процесса.

Будем считать, что возбуждено большое число поверхностных плазмон-поляритонов, так что создаваемое ими электромагнитное поле можно рассматривать классически. Пусть указанные неоднородности являются заполненными золотом впадинами на поверхности GaAs. Для дальнейших оценок примем, что эти впадины имеют сферическую форму с радиусом $r$ и расстояние от поверхности GaAs до их центров также равно $r$. Будем считать, что при вакуумной длине волны излучения $\sim 1$ мкм размер этих неоднородностей $2 r$ меньше длины волны поверхностного плазмон-поляритона, масштаба экспоненциального спадания его поля в GaAs при удалении от границы GaAs с золотом, а также длины волны обычных фотонов в GaAs. Тогда можно считать, что каждая такая неоднородность находится в однородном электрическом поле поверхностного плазмон-поляритона $\mathbf{E}$ (здесь и далее применяем комплексное представление величин, так что истинные физические величины равны удвоенным действительным частям от используемых комплексных величин) и имеет дипольный момент

$$
\mathbf{d}_{1} \equiv \frac{\varepsilon_{1}-1}{4 \pi} \frac{3 \varepsilon_{2}}{2 \varepsilon_{2}+\varepsilon_{1}} \mathbf{E} v,
$$

где $v \equiv 4 \pi r^{3} / 3$ - объем неоднородности и использовано решение известной задачи о вычислении поляризации шара в однородном внешнем электрическом поле [9]. Разность между $\mathbf{d}_{1}$ и дипольным моментом, которым обладал бы занимаемый неоднородностью объем, если бы неоднородности не было, т.е. $\mathbf{d}_{2} \equiv\left(\varepsilon_{2}-1\right) \mathbf{E} v / 4 \pi$, и есть дипольный момент $\mathbf{d}$, ответственный за рассеяние неоднородностью поверхностных плазмон-поляритонов в обычные фотоны.

Для дальнейших оценок будем пренебрегать влиянием металла на излучение неоднородностью электромагнитных волн. Тогда, используя известную формулу [8] для мощности дипольного излучения в однородной диэлектрической среде с диэлектрической проницаемостью $\varepsilon_{2}$, находим, что мощность рассеиваемого неоднородностью излучения равняется

$$
I \equiv 4|\mathbf{d}|^{2} \omega_{0}^{4} \sqrt{\varepsilon_{2}} / 3 c^{3},
$$

где, согласно сказанному выше, частота $\omega$ поверхностных плазмон-поляритонов положена равной частоте $\omega_{0}$ перехода в КТ. Далее, умножая $I$ на поверхностную 
концентрацию неоднородностей $n$, находим пространственную скорость убыли потока энергии поверхностного плазмон-поляритона через ортогональную направлению его распространения $\mathbf{k}_{\|} / k_{\|}$и имеющую единичную ширину поперек этого направления поверхность. ${ }^{3}$ Сам указанный поток энергии дается интегралом по $x$ в бесконечных пределах от усредненного по времени вектора Пойнтинга поверхностного плазмон-поляритона $\mathbf{P} \equiv c \operatorname{Re}\left[\mathbf{E}, \mathbf{B}^{*}\right] / 2 \pi$, спроецированного на $\mathbf{k}_{\|} / k_{\|}$. Для вычисления $\mathbf{E}$ и $\mathbf{B}$ можно пользоваться формулой (1) для оператора вектор-потенциала поверхностных плазмон-поляритонов в кулоновской калибровке, ограничив суммирование в ней одним $\mathbf{k}_{\|}$(т.е. применяя ее для описания одного поверхностного плазмон-поляритона с волновым вектором $\mathbf{k}_{\|}$) и помня, что в рассматриваемом классическом пределе операторы уничтожения, $\hat{a}_{\mathbf{k}_{\|}}$, и рождения, $\hat{a}_{\mathbf{k}_{\|}^{+}}^{+}$поверхностных плазмонполяритонов с волновым вектором $\mathbf{k}_{\|}$становятся комплексными числами, а следовательно, и сам оператор вектор-потенциала $\hat{\mathbf{A}}$ - вектором. В результате имеем $\mathbf{E}=-a_{\mathbf{k}_{\|}} \dot{\mathbf{A}}_{\mathbf{k}_{\|}} / c, \mathbf{B}=a_{\mathbf{k}_{\|}} \operatorname{rot} \mathbf{A}_{\mathbf{k}_{\|}}$. Деля вычисленный таким образом поток энергии на $I n$, находим длину рассеяния $l$ поверхностных плазмон-поляритонов в обычные фотоны на неоднородностях границы металл-полупроводник:

$$
l=\frac{3 \pi c^{5}\left(\varepsilon_{1}+\varepsilon_{2}\right)^{2}}{v^{2} \varepsilon_{2}\left|3 \varepsilon_{2}\left(\varepsilon_{1}-1\right) /\left(2 \varepsilon_{2}+\varepsilon_{1}\right)-\varepsilon_{2}+1\right|^{2}\left(-\varepsilon_{1}\right)^{3 / 2} \omega_{0}^{5} n} .
$$

Размер и поверхностная концентрация неоднородностей, обеспечивающие преобразование в обычные фотоны поверхностных плазмон-поляритонов, возбуждаемых практически всеми КТ, находятся из следующих соображений. Преобразование поверхностных плазмонполяритонов в обычные фотоны на неоднородностях будет практически полным, если соответствующая длина рассеяния $l$ будет существенно меньше длины поглощения поверхностного плазмон-поляритона. Поэтому выберем $l \approx 2.3$ мкм, что более чем в 3 раза меньше, чем длина поглощения поверхностного плазмон-поляритона $1 /\left(2 \operatorname{Im} k_{\|}\right) \approx 7$ мкм для вакуумной длины волны излучения 1 мкм. Поскольку $l \propto v^{-2} n^{-1} \propto(2 r)^{-6} n^{-1}$, то для достижения указанного значения $l$ при минимально возможной $n$ размер неоднородностей $2 r$ должен быть

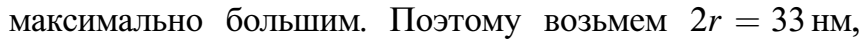
т. е. максимальную величину, при которой еще выполняются использованные при выводе (6) предположения об однородности поля поверхностного плазмон-поляритона в пределах неоднородности, т.е. о малости $2 r$ по сравнению с длиной волны поверхностного плазмонполяритона $\left(2 \pi / k_{\|} \approx 249\right.$ нм $)$ и масштабом спадания его поля в $\mathrm{GaAs}$ при удалении от границы с золотом $\left(-1 / \operatorname{Im} k_{x 2} \approx 81 \mathrm{Hм}\right)$, а также о справедливости дипольного приближения при вычислении мощности рассеива-

\footnotetext{
3 Здесь мы пренебрегаем относительно малой мнимой частью $\mathbf{k}_{\|}$, считая его действительным.
}

емого неоднородностью излучения, т. е. о малости $2 r$ по сравнению с длиной волны обычных фотонов в $\mathrm{GaAs}$ $(285 \mathrm{Hм})$. Тогда $n=10^{10} \mathrm{~cm}^{-2}$, что вполне достижимо экспериментально.

Заметим еще, что неоднородности в виде бугорков на поверхности полупроводника, окруженных металлом, рассеивают поверхностные плазмон-поляритоны в обычные фотоны значительно слабее, чем рассмотренные неоднородности в виде впадин в полупроводнике, заполненных металлом. Это связано с тем, что неоднородности в виде бугорков излучают фотоны в основном не в полупроводник, а в металл, где они вследствие отрицательности диэлектрической проницаемости металла распространяться не могут.

В заключение данного раздела следует также отметить, что эксперименты, подтверждающие принципиальную возможность преобразования поверхностных плазмон-поляритонов в фотоны на неоднородностях гетерограницы металл-полупроводник, проводились нами ранее. В них измерялась интенсивность фотолюминесценции наногетероструктур с КТ, покрытых полупрозрачными пленками золота. Было установлено, что для наногетероструктур, в которых граница металл-полупроводник (по данным исследования с помощью микроскопа) характеризовалась малой поверхностной концентрацией неоднородностей, интенсивность фотолюминесценции была значительно ниже, чем для наногетероструктур, в которых граница металл-полупроводник была сильно неоднородной. Объяснение этого факта, согласно сказанному выше, состоит в том, что в наногетероструктурах с однородной границей металл-полупроводник превращение поверхностных плазмон-поляритонов в обычные фотоны, свободно выходящие в окружающее пространство, возможно лишь на торцах структуры. Поэтому претерпевать такое превращение могут лишь поверхностные плазмон-поляритоны, возбужденные КТ, удаленными от торцов на расстояния не больше длины поглощения поверхностных плазмонполяритонов за счет омической диссипации в металле ( 7 мкм, см. выше). В то же время в наногетероструктурах с неоднородной границей металл-полупроводник поверхностные плазмон-поляритоны, возбужденные КТ, находящимися на любом удалении от торцов, могут превратиться на неоднородностях этой границы в обычные фотоны, которые затем свободно выйдут в окружающее пространство. Поскольку латеральные размеры структуры значительно превышают длину поглощения поверхностных плазмон-поляритонов за счет омической диссипации в металле, это означает, что интенсивность фотолюминесценции наногетероструктур с неоднородной границей металл-полупроводник должна быть значительно выше, чем наногетероструктур с однородной границей, что и наблюдалось в эксперименте. Однако в проведенных экспериментах размеры неоднородностей границы металл-полупроводник и их поверхностная концентрация не были равны рассчитанным в данном разделе значениям, обеспечивающим преобразование в 
обычные фотоны поверхностных плазмон-поляритонов, возбуждаемых практически всеми КТ. Создание наногетероструктур с найденными значениями этих параметров и измерение эффективности преобразования в них поверхностных плазмон-поляритонов в обычные фотоны является задачей будущих работ.

\section{4. Заключение}

Таким образом, в настоящей работе найдены улучшенные параметры гетероструктур с квантовыми точками InAs в матрице GaAs для создания на их основе быстродействующих и имеющих высокий коэффициент полезного действия светодиодов Шоттки ближнего инфракрасного диапазона частот. Такие устройства являются востребованными в телекоммуникационных и информационных приложениях.

Исследование выполнено при финансовой поддержке РФФИ в рамках научного проекта № 16-02-00450 а.

\section{Список литературы}

[1] A. Chahboun, M.I. Vasilevskiy, N.V. Baidus, A. Cavaco, N.A. Sobolev, M.C. Carmo, E. Alves, B.N. Zvonkov. J. Appl. Phys., 103 (8), 083548 (2008).

[2] С.А. Майер. Плазмоника: теория и приложения (Москва, Ижевск, R\&C Dynamics, 2011) гл. 2.

[3] E.M. Purcell. Phys. Rev., 69 (11-12), 681 (1946).

[4] J. Kalkman, H. Gersen, L. Kuipers, A. Polman. Phys. Rev. B, 73 (7), 075317 (2006).

[5] N.V. Baidus, B.N. Zvonkov, P.B. Mokeeva, E.A. Uskova, S.V. Tikhov, M.I. Vasilevskiy, M.J.M. Gomes, S.A. Filonovich. Semicond. Sci. Technol., 19 (4), S469 (2004).

[6] Н. Ашкрофт, Н. Мермин. Физика твердого тела (М., Мир, 1979) гл. 1, 2.

[7] D.J. Bergman, M.I. Stockman. Phys. Rev. Lett., 90 (2), 027402 (2003).

[8] В.Л. Гинзбург. Теоретическая физика и астрофизика (М., Физматлит, 1987) гл. 6.

[9] Л.Д. Ландау, Е.М. Лифшиц. Электродинамика сплошных сред (М., Физматлит, 2001) гл. 9.

[10] В.Б. Берестецкий, Е.М. Лифшиц, Л.П. Питаевский. Квантовая электродинамика (М., Физматлит, 2002) гл. 1, 6.

[11] Н.В. Байдусь, А.Н. Яблонский. Тр. ХІХ Межжунар. симп. „Нанофизика и наноэлектроника“ (Н. Новгород, 2015) (Изд-во Нижегородского гос. ун-та им. Н. И. Лобачевского, 2015) т. 2, с. 429.

[12] M. Usman, S. Heck, E. Clarke, P. Spencer, H. Ryu, R. Murray, G. Klimeck. J. Appl. Phys., 109 (10), 104510 (2011).

\section{Nanoheterostructures with improved characteristics for high-speed and efficient plasmon-polariton light emitting Schottky diodes}

\author{
N.V. Baidus ${ }^{+*}$, V.A. Kukushkin ${ }^{+}$, B.N. Zvonkov*, \\ S.M. Nekorkin*
}

+ Nizhny Novgorod State University

named after N.I. Lobachevsky,

603950 Nizhny Novgorod, Russia

* Research Physico-Technical Institute

of Nizhny Novgorod State University

named after N.I. Lobachevsky,

603950 Nizhny Novgorod, Russia

$¥$ Institute of Applied Physics,

Russian Academy of Sciences,

603950 Nizhny Novgorod, Russia

Abstract By means of experimental and theoretical approaches we found parameters of heterostructures with quantum dots InAs in GaAs matrix providing the creation on such structures of high-speed and efficient plasmon-polariton near infrared light emitting Schottky diodes. Quantum dots shoud be placed on a heavy doped (up to the doping impurity concentration $10^{19} \mathrm{~cm}^{-3}$ ) GaAs buffer layer and separated from a metal by a thin (with thickness $10-30 \mathrm{~nm}$ ) undoped GaAs cover layer. The interface between metal (e.g. $\mathrm{Au}$ ) and $\mathrm{GaAs}$ will provide the efficient scattering of surface plasmon-polaritons into usual photons if it has inhomogeneities in the form of metal-filled cavities in GaAs with characteristic dimension $30 \mathrm{~nm}$ and surface concentration $10^{10} \mathrm{~cm}^{-2}$.

Редактор Л.В. Шаронова 